\title{
Comprehensive Analysis Suggests Overlapping Expression of Rice ONAC Transcription Factors in Abiotic and Biotic Stress Responses
}

\author{
Lijun Sun ${ }^{1,2}$, Lei Huang ${ }^{1}$, Yongbo Hong ${ }^{1}$, Huijuan Zhang ${ }^{1}$, Fengming Song ${ }^{1}$ \\ and Dayong $\mathrm{Li}^{1, *}$
}

1 National Key Laboratory for Rice Biology, Institute of Biotechnology, Zhejiang University, Hangzhou, Zhejiang 310029, China;

E-Mails: slj.1226@163.com (L.S.); leihero2008@163.com (L.H.); yongbohong@126.com (Y.H.); zhanghj82@zju.edu.cn (H.Z.); fmsong@zju.edu.cn (F.S.)

2 Institute of Analytical Chemistry for Life Science, School of Public Health, Nantong University, Nantong 226019, China

* Author to whom correspondence should be addressed; E-Mail: dyli@zju.edu.cn; Tel./Fax: +86-571-8898-2271.

Academic Editor: Marcello Iriti

Received: 29 December 2014 / Accepted: 11 February 2015 / Published: 17 February 2015

\begin{abstract}
NAC (NAM/ $\underline{\mathrm{ATAF}} \underline{\mathrm{CUC}}$ ) transcription factors comprise a large plant-specific gene family that contains more than 149 members in rice. Extensive studies have revealed that NAC transcription factors not only play important roles in plant growth and development, but also have functions in regulation of responses to biotic and abiotic stresses. However, biological functions for most of the members in the NAC family remain unknown. In this study, microarray data analyses revealed that a total of 63 ONAC genes exhibited overlapping expression patterns in rice under various abiotic (salt, drought, and cold) and biotic (infection by fungal, bacterial, viral pathogens, and parasitic plants) stresses. Thirty-eight $O N A C$ genes exhibited overlapping expression in response to any two abiotic stresses, among which 16 of 30 selected $O N A C$ genes were upregulated in response to exogenous ABA. Sixty-five $O N A C$ genes showed overlapping expression patterns in response to any two biotic stresses. Results from the present study suggested that members of the ONAC genes with overlapping expression pattern may have pleiotropic biological functions in regulation of defense response against different abiotic and biotic stresses, which provide clues for further functional analysis of the $O N A C$ genes in stress tolerance and pathogen resistance.
\end{abstract}


Keywords: NAC transcription factors; overlapping expression; rice; abiotic and biotic stress

\section{Introduction}

Plants are always subject to various types of abiotic (e.g., high-salinity, drought, and cold) and biotic (infection by fungal, bacterial and viral pathogens, and infestation by herbivores and parasitic plants) stresses under variable environmental conditions, and have developed a series of mechanisms at physiological, biochemical, and molecular levels to combat these stresses [1-5]. In response to abiotic and biotic stresses, plants often activate a battery of defense responses that include inducible expression of a set of stress-related genes, which are regulated directly or indirectly by different types of transcription factors. Previous studies have showed that dozens of transcription factors belonging to the ERF, MYB, WRKY, and bZIP families are involved in regulation of plant stress responses [6-9].

The NAC (NAM/ $\underline{\mathrm{A} T A F} / \underline{\mathrm{CUC}})$ transcription factor family, as one of the largest plant-specific gene families, was first reported in 1997 [10]. NAC proteins are characterized with a conserved region at their $N$-terminal ends and a highly diverged $C$-terminus [11,12]. Genome-based bioinformatics analyses have showed that NAC proteins comprise a large family with more than 100 members in Arabidopsis, rice, tobacco, soybean, and populus [13-18]. However, only a few of them have been functionally characterized for their biological functions. To date, the members of the $N A C$ gene family have been demonstrated to play important roles in diverse biological processes of plant growth and development, e.g., cell wall biosynthesis and senescence (for reviews, see [19,20]).

NAC transcription factors have been also demonstrated to play important roles in regulating plant abiotic and biotic stress responses. Transcriptional profiling analysis revealed that at least $33 N A C$ genes were responsible to abiotic stress, including high salt in Arabidopsis [21]. It was further showed that expression of Arabidopsis ANAC019, ANAC055, and ANAC072/RD26 was induced by different abiotic stresses including drought and high salt, and overexpression of ANAC019, ANAC055, $A N A C 072 / R D 26$, or $A T A F 1$ significantly increased drought tolerance [22,23]. Furthermore, transgenic Arabidopsis plants overexpressing $A N A C 072 / R D 26$ were highly sensitive to ABA [24]. In rice, overexpression of stress-responsive SNAC1 or SNAC2 (OSNAC6) enhanced drought and salinity tolerance [25-28], and the $S N A C 1$-overexpressing transgenic rice showed increasing drought tolerance in the field under severe drought stress conditions at the reproductive stage without any phenotypic changes or adverse effect on yields [25]. OsNAC5 as a transcriptional activator interacts with OsNAC6 and SNAC1 and overexpression of OSNAC5 resulted in improved tolerance to high salt as compared to control plants [29]. Similarly, overexpression of OsNAC10 (ONAC122) or OsNAC045 in rice increased drought and salt tolerance [30,31]. Furthermore, chickpea $N A C$ genes such as CarNAC1, CarNAC3, and CarNAC5 and tomato NAC genes like SINAC1, SINAM1, and SINAC2 were also reported to be involved in abiotic stress responses [32-36].

Accumulating evidence has implied that the $N A C$ genes play important roles in plant defense response against pathogen infection. The Arabidopsis ATAF1 and ATAF2 genes have been demonstrated through knockout or overexpression approaches to be negative regulators of defense response against different pathogens, e.g., Botrytis cinerea, Pseudomonas syringae pv. tomato, Alternaria brassicicola, 
or Fusarium oxysporum [23,37,38]. Barley HvNAC6, an ATAF1 homologous gene, was shown to contribute to penetration resistance against Blumeria graminis f. sp. hordei [39]. In rice, three ONAC genes have been demonstrated to have function in disease resistance response. OsNAC6 was induced by Magnaporthe grisea, the causal agent of blast disease, and overexpression of OsNAC6 in rice resulted in improved blast resistance [28]. OsNAC4 was shown to be involved in regulation of hypersensitive cell death because overexpression of OSNAC4 led to extensive HR cell death, while knockdown of OsNAC4 suppressed HR cell death in response to an avirulent bacterial pathogen [40]. RIM1 (ONAC054) was revealed to be a specific $N A C$ gene that negatively regulates defense response against rice dwarf virus (RDV) [41]. A Tos17-inserted mutant line showed no typical disease symptoms upon RDV infection but remained susceptible to other viral pathogens [41]. Other $N A C$ genes such as Arabidopsis TIP, wheat GRAB1/GRAB2, and tomato SINAC1 have also been reported to be involved in plant-virus interactions, probably through interacting with virus proteins [42-44]. It was also found that pepper $C a N A C 1$, wheat TaNAC4, and soybean GmNAC6 are pathogen responsible, suggesting their possible involvement in defense response to pathogen infection [45-47]; however, further functional characterization is required for these pathogen-responsive $N A C$ genes to clarify their function in plant disease resistance response.

Although several ONAC genes such as SNAC1, OsNAC6, OsNAC5, OsNAC10, and OsNAC4 have been characterized functionally in rice, the biological functions of most members in this family remain unknown. To extend our understanding of the biological function of the rice NAC family, we performed overlapping expression analysis using public microarray data and identified a total of 63 ONAC (following the nomenclature as suggested by Fang et al. [14]) genes that showed overlapping expression patterns under different stresses. The phylogenetic relationships suggested that the $O N A C$ genes showing overlapping expression patterns under stress conditions can be classed into 10 groups. We also isolated 15 putative stress-responsive $O N A C$ genes and analyzed their expression patterns in response to different abiotic and biotic stresses. Our results suggested that some members of the rice ONAC family may have overlapping functions in regulation of defense response against different abiotic and biotic stresses, and also provided clues for further functional analysis of ONAC genes in stress tolerance and pathogen resistance.

\section{Results}

\subsection{Inducibility and Overlapping Expression of ONAC Genes in Response to Abiotic Stresses}

Salt, drought, and cold are common stresses that cause adverse effects on the growth and development of plants and the productivity of crops [48]. To study the possible involvement of ONAC genes in response to various abiotic stresses, we mined microarray data (GSE6901) deposited in a public database [49] and performed systematic analysis for their expression patterns under salinity, drought, and cold conditions. Differential expression of the $O N A C$ genes in rice seedlings treated with different abiotic stresses such as salinity, drought, and cold conditions were analyzed by comparison with mock-treated seedlings. For drought stress, 43 ONAC genes (33 upregulated and 10 downregulated) were differentially expressed in cv. IR64 seedlings after drought treatment for $3 \mathrm{~h}$. For salt stress, 41 ONAC genes (31 upregulated and 10 downregulated) were differentially expressed in cv. IR64 seedlings 
after salt treatment for $3 \mathrm{~h}$. For cold stress, 36 ONAC genes (27 upregulated and 9 downregulated) were differentially expressed in rice seedlings under cold stress (Supplementary file 1). Further analysis of the overlapping expression patterns identified a total of 38 ONAC genes that were differentially expressed under at least two abiotic stress conditions based on analysis of microarray data GSE6901. Among these, 15 ONAC genes (SNAC1, OsNAC4, OSNAC5, OsNAC6, OsNAC8, ONAC026, ONAC034, ONAC069, ONAC092, ONAC095, ONAC096, ONAC108, ONAC122, ONAC123, and $O N A C 132)$ were upregulated and $2 O N A C$ genes (ONAC109 and ONAC117) were downregulated under drought, salt, and cold stress conditions (Figure 1A,B and Supplementary file 2). To confirm the results obtained from microarray data analysis, we analyzed by quantitative RT-PCR the expression levels of 30 selected $O N A C$ genes in response to drought, salt, and cold stresses and the results showed that the expression patterns of these $O N A C$ genes under drought, salt, and cold stress conditions were identical to the patterns obtained from the rice microarray data analysis (Figure 2C). However, the expression patterns for ONACO16 and ONAC023 were not identical (Figure 1C). Results from our overlapping expression and quantitative RT-PCR analyses are in accordance with previous studies in which genes such as SNAC1, SNAC2/OSNAC6, OSNAC5, OSNAC10, and ONAC45 were induced by different stresses including drought, high salinity, and cold stresses [25-31].

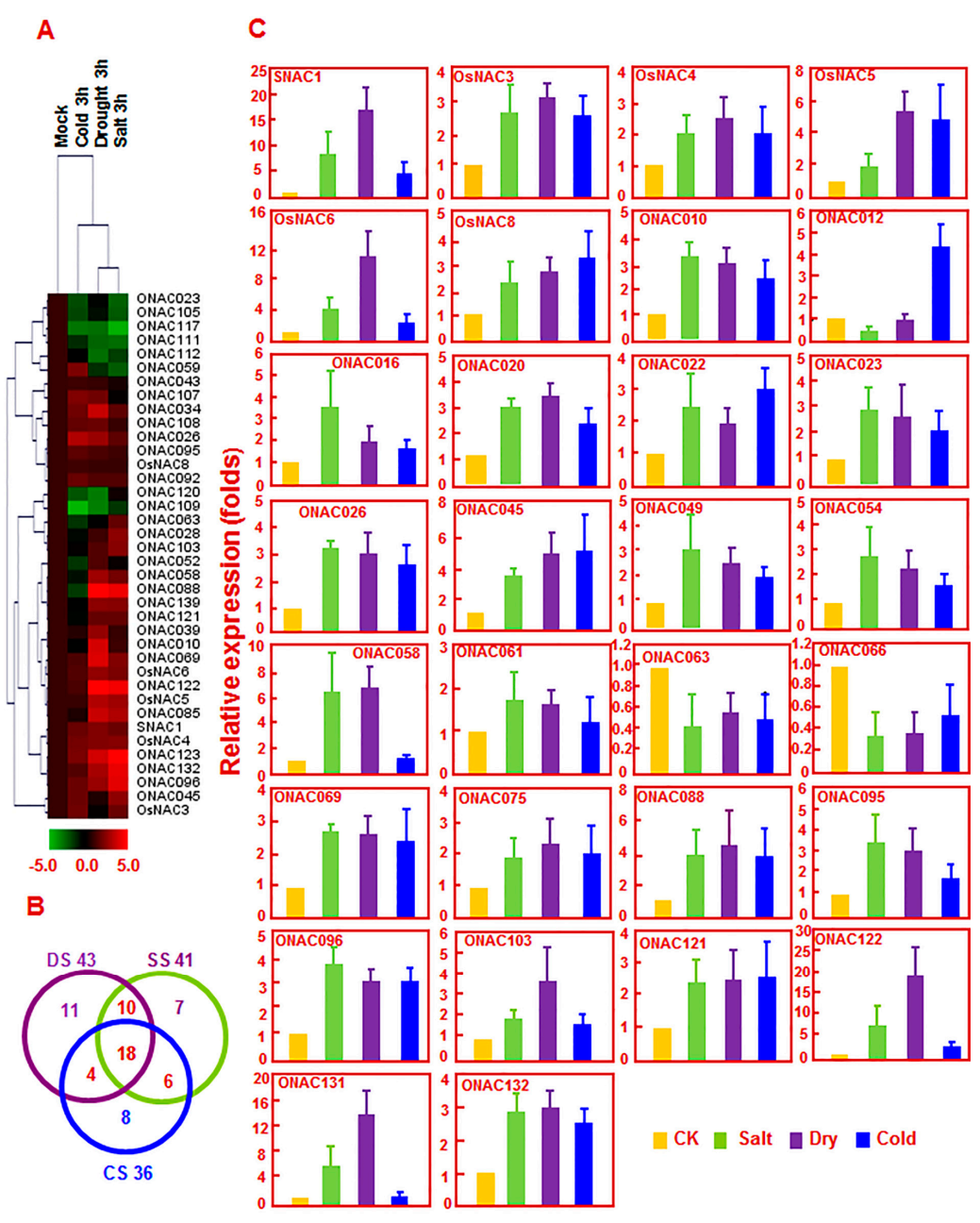

Figure 1. Overlapping expression of $O N A C$ genes in response to various abiotic stress conditions. (A) Heat map of $O N A C$ genes showing differential expression patterns under at least two abiotic stress conditions. The change values in treated samples $v s$. corresponding 
control, shown as $\left(\log _{2}(\right.$ signal intensity in treatment/signal intensity in control $)=\log _{2}{ }^{\text {signal intensity in treatment }-}$ $\log _{2}$ signal intensity in control), were used for Treeview (Supplementary file 2). The color scale of change values is shown at the bottom; (B) Venn diagram represents number of $O N A C$ genes expressed commonly or specifically under given abiotic stress conditions ( $t$-test $p<0.01$ ); (C) Differential expression of 30 selected $O N A C$ genes under drought, salt, and cold conditions, as analyzed by qPCR. Relative expression of each $O N A C$ gene was calculated by comparison with corresponding control. Error bars represent standard errors of the means from three independent biological replicates.

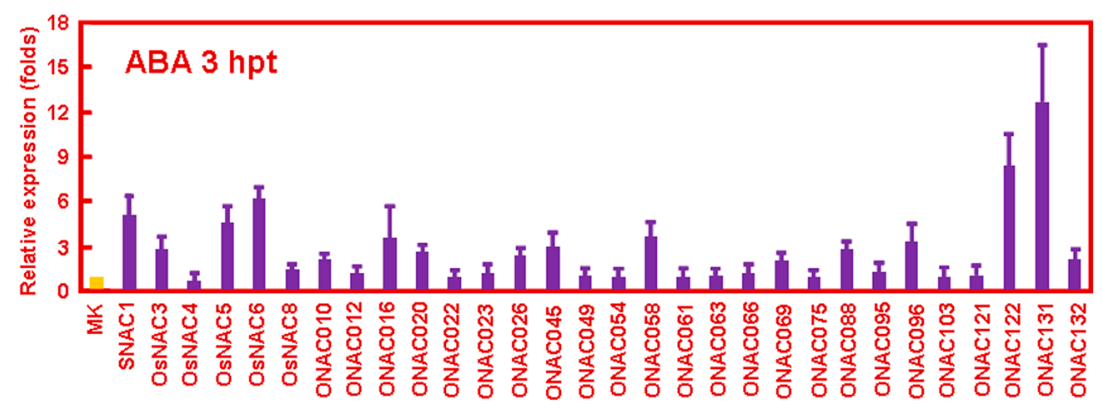

Figure 2. Differential expressions of 30 selected $O N A C$ genes in response to ABA. Rice seedlings were treated with $\mathrm{ABA}$ for $3 \mathrm{~h}$ and expression of $O N A C$ genes was analyzed by quantitative RT-PCR. Relative expression for each $O N A C$ gene was calculated by comparison with corresponding control. Error bars represent standard errors of the means from three independent biological replicates.

$\mathrm{ABA}$ is a key mediator regulating plant response to a number of abiotic stresses such as drought, high salinity, and cold through activation of many stress-related genes [50]. On the other hand, ABA has also been demonstrated to play important roles in the regulation of plant disease resistance response [50,51]. Thus, we analyzed the expression of these representative $O N A C$ genes in response to ABA treatment. Our data showed that the expression levels of these 16 ONAC genes, including SNAC1, OsNAC3, OsNAC5, OsNAC6, ONAC010, ONAC016, ONAC020, ONAC026, ONAC045, ONAC058, ONAC069, ONAC088, ONAC095, ONAC122, ONAC131, and ONAC132 were upregulated in rice seedlings at $3 \mathrm{~h}$ after ABA treatment (Figure 2).

\subsection{Inducibility and Overlapping Expression of ONAC Genes in Response to Biotic Stress}

To explore possible involvement of $O N A C$ genes in rice response to biotic stress, we analyzed the expression of the $O N A C$ genes in rice seedlings after infection with different types of pathogens or infestation by parasitic plants.

Rice blast disease, caused by M. oryzae, is one of the most serious and widespread diseases of rice. Due to the agronomic importance of rice, it is important to understand the molecular mechanisms of disease resistance against $M$. oryzae. We first analyzed the expression patterns of ONAC genes by mining the microarray data GSE7256 (leaves of 14-day-old rice seedlings infected with a virulent strain FR13 of $M$. grisea) and GSE18361 (roots of rice seedlings infected with by strain Guy11 of M. oryzae) [52,53]. For leaf infection by strain FR13, a total of 75 ONAC genes that were differentially 
expressed at 3 and 4 days post-inoculation (DPI) were identified. Among them, 23 and 17 ONAC genes were upregulated and downregulated, respectively, at 3 DPI, whereas 42 and 21 ONAC genes were upregulated and downregulated, respectively, at 4 DPI (Supplementary file 1). Fifteen $O N A C$ genes (ONAC1, OsNAC3, OsNAC4, OsNAC8, ONAC010, ONAC012, ONAC017, ONAC034, ONAC039, ONAC059, ONAC085, ONAC095, ONAC096, ONAC132, and ONAC134) were upregulated while 5 ONAC genes (ONAC016, ONAC052, ONAC079, ONAC094, and ONAC138) were downregulated at 3 and 4 DPI. For root infection by Guy11, a total of $64 O N A C$ genes were found to be differentially expressed at 2, 4, and 6 DPI. Among them, expression of 24, 19, and 19 ONAC genes at 2, 4, and 6 DPI, respectively, was upregulated, whereas expression of 17, 9, and $14 O N A C$ genes at 2, 4, and 6 DPI, respectively, was downregulated (Supplementary file 1). In total, 88 ONAC genes were differentially expressed after rice infection by $M$. grisea and 50 ONAC genes exhibited overlapping expression patterns between leaf and root infection by $M$. grisea (Figure 3A and Supplementary file 2). To confirm the results obtained from microarray data analysis, we analyzed by quantitative RT-PCR the expression levels of 30 selected $O N A C$ genes in rice seedlings after leaf infection with $M$. grisea and our results showed that the expression patterns of these $O N A C$ genes in response to infection of $M$. grisea at 3 and 4 DPI were similar to those obtained from microarray data analysis (Figure 3B). Among these $O N A C$ genes that showed differential expression in response to $M$. oryzae, OsNAC6 and $S N A C 1 / O S N A C 19$ were previously reported to be induced by $M$. grisea $[28,54]$.

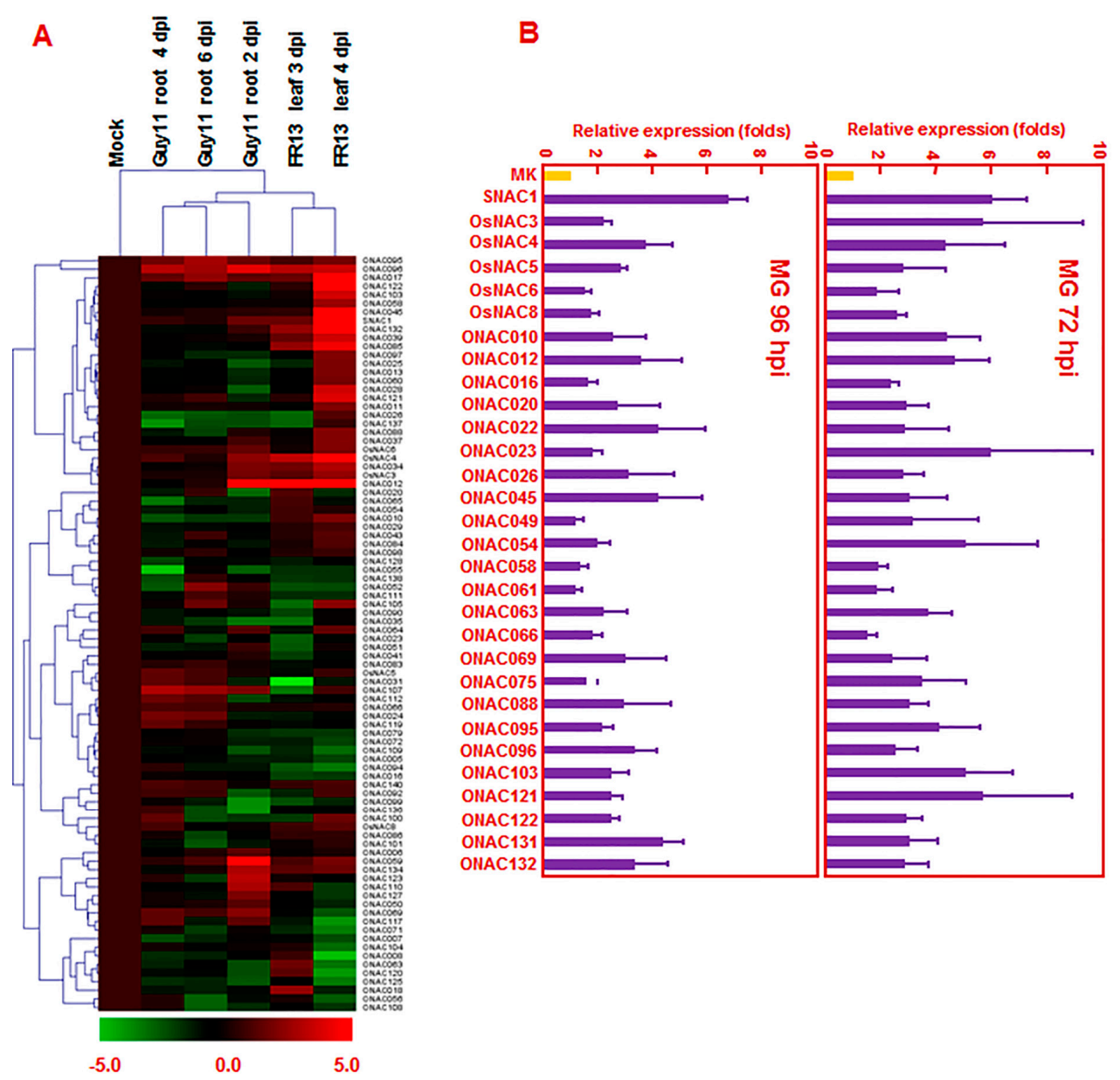

Figure 3. Differential expression of $O N A C$ genes in response to infection by $M$. oryzae. (A) Heat map of ONAC genes showing differential expression patterns after leaf and root 
infection by $M$. oryzae. The change values in treated samples vs. corresponding control, shown as $\left(\log _{2}\right.$ (signal intensity in treatment/signal intensity in control) $=\log _{2}$ signal intensity in treatment $\log _{2}$ signal intensity in control), were used for Treeview (Supplementary file 2). The color scale of change values is shown at the bottom; (B) Expression of 30 selected ONAC genes in response to infection by $M$. oryzae. Quantitative RT-PCR analysis was performed for samples of the leaves of 2-week-old Yuanfengzao collected at 72 and $96 \mathrm{~h}$ after inoculation with $M$. oryzae. Relative expression of each $O N A C$ gene was calculated by comparison with corresponding control. Error bars represent standard errors of the means from three independent biological replicates.

Bacterial blight caused by Xanthomonas oryzae pv. oryzae (Xoo) and bacterial leaf streak caused by $X$. oryzae pv. oryzicola $(X o c)$ are two major rice bacterial diseases with distinctive types of infection style. Xoo typically invades the xylem system, whereas Xoc generally infects and colonizes the mesophyll parenchyma. Previously, the abundance of transcripts in 14-day-old cv. Nipponbare seedlings at 2, 4, 8, 24, and $96 \mathrm{~h}$ after inoculation with Xoo strain PXO99A and Xoc strain BLS256 was analyzed (microarray data set GSE16793) to establish the global structure of susceptible responses to infection by $X o o$ and $X o c$ [55]. We used these data sets and analyzed the expression profiling of the $O N A C$ genes' response to infection with Xoo and Xoc. Our analyses showed that 5, 3, 7, 8, and $8 O N A C$ genes were upregulated and 6, 7, 2, 10, and $4 O N A C$ genes were downregulated at 2, 4, 8, 24, and $96 \mathrm{~h}$, respectively, after inoculation with $X o o$ strains PXO99A; and that 10, 8, 9, 6, and 8 ONAC genes were upregulated and 6,8,5,3, and $9 O N A C$ genes were downregulated at 2, 4, 8, 24, and $96 \mathrm{~h}$, respectively, after inoculation with $X O c$ strain BLS256 (Supplementary file 1). Importantly, a total of $28 O N A C$ genes were found to exhibit overlapping expression patterns in rice plants after infection with $X O O$ and Xoc. Among these, 13 ONAC genes (ONAC012, ONAC18, ONAC025, ONAC065, ONAC069, ONAC083, ONAC099, ONAC108, ONAC112, ONAC117, ONAC132, ONAC136, and ONAC140) were upregulated and 10 ONAC genes (ONAC020, ONAC023, ONAC056, ONAC076, ONAC100, ONAC110, ONAC111, ONAC125, ONAC135, and ONAC137) were downregulated in both Xoo- and $X o c$-infected rice plants (Figure 4A and Supplementary file 2).

Rice stripe disease, caused by rice stripe virus (RSV), which is transmitted by small brown planthoppers (Laodelphax striatellus), is one of the most serious viral diseases and causes severe yield loss in rice. Transcriptional profiling (microarray data set GSE11025) was performed using Affymetrix rice genome arrays and investigated the molecular differences in subspecies cv. WuYun3 and cv. KT95-418 in response to RSV infection (9-day old seedlings inoculated by exposing to approximately 100 viruliferous adults of $L$. striatellus for 2 days and then transplanted to an insect-free greenhouse at $25 \pm 3{ }^{\circ} \mathrm{C}$ ) [56]. Analyses of the expression patterns of $O N A C$ genes in response to RSV using these microarray data resulted in identification of at least $27 O N A C$ genes that were upregulated and 16 genes were downregulated in cv. WuYun3, while 22 ONAC genes were upregulated and 19 genes were downregulated in cv. KT95-418 in response to RSV infection (Supplementary file 1). A total of $17 O N A C$ genes showed overlapping expression patterns in cv. WuYun3 and cv. KT95-418 plants after RSV infection. Among them, 7 ONAC genes (ONAC012, ONAC17, ONAC034, ONAC035, ONAC095, ONAC134, and ONAC135) were upregulated and 6 ONAC genes (ONAC025, ONAC28, ONAC099, $O N A C 103, O N A C 109$, and $O N A C 122$ ) were downregulated (Figure 4B). We analyzed by quantitative 
RT-PCR the expression levels of 30 selected $O N A C$ genes in rice seedlings after RSV infection and our results showed that the expression patterns of these $O N A C$ genes in response to RSV were similar to those obtained from overlapping expression analysis using public microarray data (Figure 4C).

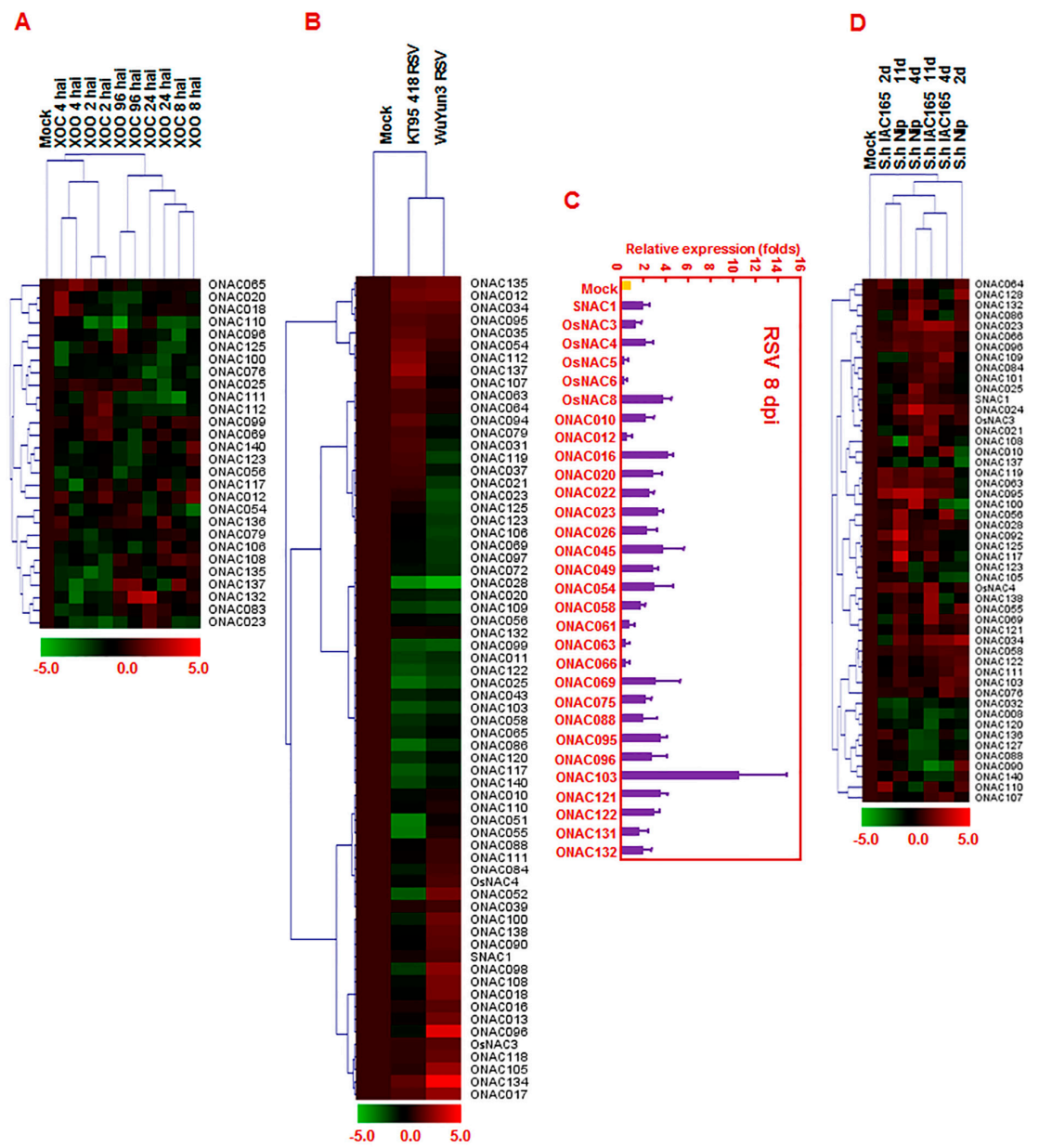

Figure 4. Differential expressions of $O N A C$ genes in response to $X o o, X o c$ and RSV. $(\mathbf{A}, \mathbf{B})$ Heat maps of $O N A C$ genes showing differential expression patterns in rice seedlings after $X o o$ and $X o c$ infection and RSV infection, respectively. The change values in treated samples $v$. corresponding control, shown as $\left(\log _{2}\right.$ (signal intensity in treatment/signal intensity in control) $=$ $\log _{2}$ signal intensity in treatment $-\log _{2}$ signal intensity in control), were used for Treeview (Supplementary file 2). The color scale of change values is shown at the bottom; (C) Differential expression of 30 selected $O N A C$ genes in rice after RSV infection; (D) Heat Map of $O N A C$ genes showing differential expression patterns after infestation by $S$. hemonthica. Quantitative RT-PCR analysis was performed and relative expression for each ONAC gene was calculated by comparison with corresponding control. Error bars represent standard errors of the means from three independent biological replicates.

Striga hermonthica is a kind of root parasitic plant of rice and causes devastating loss of rice yield. Transcriptional profiling was performed to identify the possible molecular mechanism underlying susceptible and resistant responses of rice plants of different cultivars, where cv. Nipponbare is resistant and cv. IAC165 is susceptible, respectively, to infestation by $S$. hermonthica (three-week-old cv. Nipponbare and cv. IAC165 seedling infested with $25 \mathrm{mg}$ of preconditioned and pregerminated 
seeds of S. hermonthica and root samples harvested at 2, 4, and 11 DPI) [57]. We mined the microarray data from the above-mentioned study and analyzed the expression patterns of $O N A C$ genes in response to infestation by $S$. hermonthica. Our analyses revealed that 9,24 , and $30 O N A C$ genes were upregulated and 2, 9, and $9 O N A C$ genes were downregulated in cv. IAC165 at 2, 4, and 11 DPI, while 18,28, and $32 O N A C$ genes were upregulated and 5, 7, and $5 O N A C$ genes were downregulated in cv. Nipponbare after infestation with $S$. hermonthica (Supplementary file 1). A total of 49 ONAC genes exhibited overlapping expression patterns in plants of cv. IAC165 and cv. Nipponbare after infection by $S$. hermonthica (Figure 4D and Supplementary file 2). Interestingly, the number of the $O N A C$ genes with upregulated expression patterns was significantly more than that of the $O N A C$ genes with downregulated expression patterns in response to $S$. hermonthica both in cv. IAC165 and cv. Nipponbare after infection by $S$. hermonthica, suggesting that most of the $O N A C$ genes can be indeced by $S$. hermonthica infection.

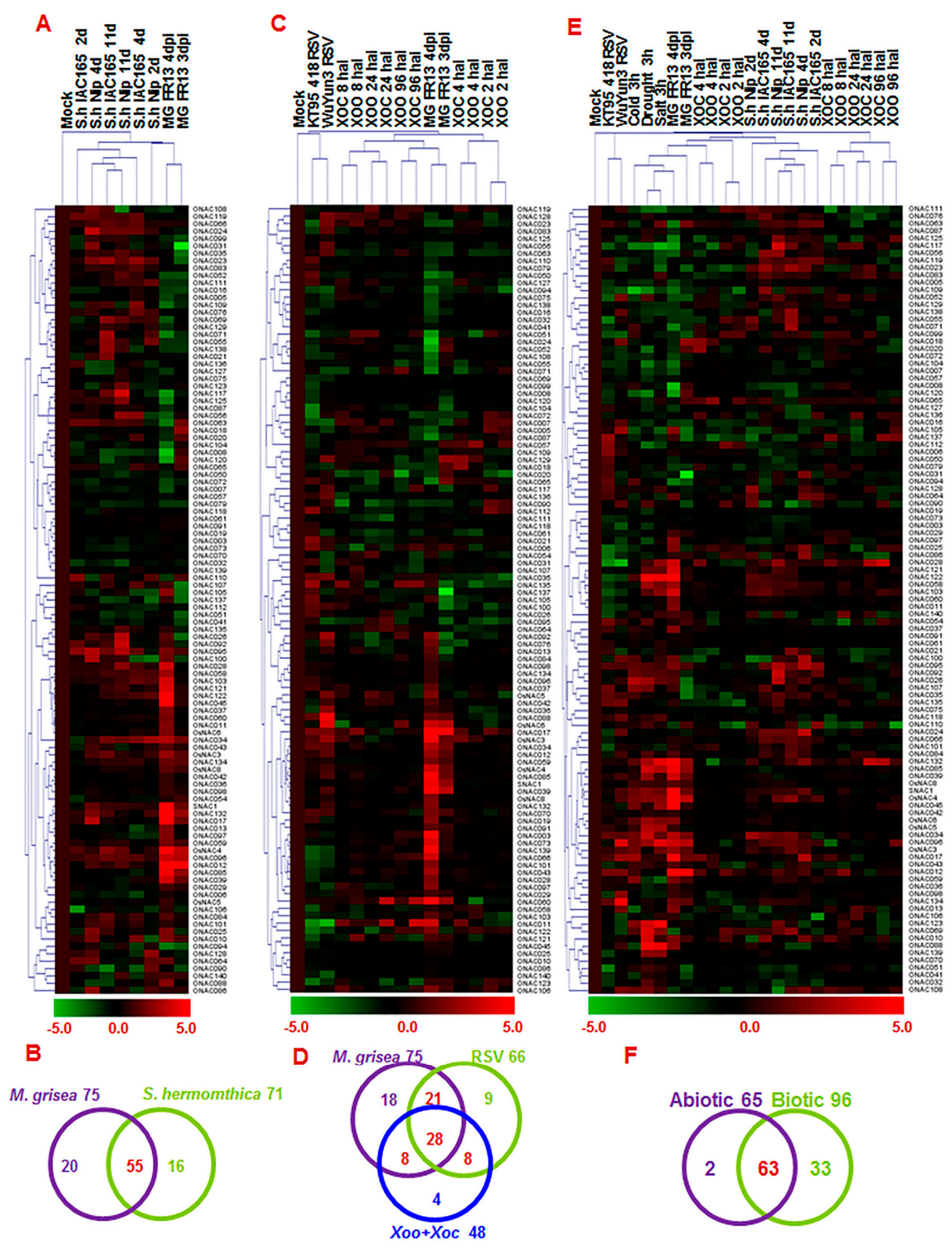

Figure 5. Overlapping expression of $O N A C$ genes in response to various biotic and abiotic stresses. (A) Heat map of $O N A C$ genes, showing differential expression patterns in 
response to $M$. oryzae and $S$. hermonthica; (B) Venn diagram represents number of ONAC genes expressed commonly or specifically in response to $M$. oryzae and S. hermonthica $(t$-test $p<0.01)$; (C) Heat map of $O N A C$ genes showing differential expression patterns in response to $M$. oryzae, RSV, Xoo, and Xoc; (D) Venn diagram represents number of $O N A C$ genes expressed commonly or specifically in response to M. oryzae, RSV, Xoo, and Xoc $(t$-test $p<0.01)$; (E) Heat map of $O N A C$ genes showing differential expression patterns under abiotic (salt, drought, and cold) and biotic (infection by M. oryzae, Xоo, Хoc, or RSV, or infestation by $S$. hermonthica) stress; (F) Venn diagram represents number of $O N A C$ genes expressed commonly or specifically in response to biotic and abiotic stresses $(t$-test $p<0.01)$. The change values $(\mathbf{A}, \mathbf{C}, \mathbf{E})$ in treated samples $v s$. corresponding control, shown as $\left(\log _{2}\right.$ (signal intensity in treatment/signal intensity in control) $=\log _{2}$ signal intensity in treatment $\log _{2}$ signal intensity in control), and the relative expression folds were calculated by $2^{\text {change values }}$. Both of them were used for Treeview (Supplementary file 2). The color scales of change values and relative expression folds were shown at the bottom.

\subsection{Overlapping Expression of Different Groups of ONAC Genes in Response to Abiotic and} Biotic Stresses

When comparing the expression patterns of the $O N A C$ genes in response to different biotic stresses, we identified 51 ONAC genes with overlapping expression patterns in responses to infection by $M$. oryzae and to infestation by S. hermonthica. Among these, 22 ONAC genes were upregulated in response to M. oryzae and S. hermonthica (Figure 5A,B and Supplementary file 2). Similarly, 28 ONAC genes exhibited overlapping expression patterns in response to infection by different type of pathogens including M. oryzae, RSV, Xoo, and Xoc (Figure 5D), and at least 37 ONAC genes showed similar expression patterns in response to infection by any two different pathogens (Figure 5C and Supplementary file 2).

Overlapping expression patterns of the $O N A C$ genes in response to different abiotic and biotic stresses were analyzed further. In total, $65 O N A C$ genes responsive to abiotic stress and 96 ONAC genes responsive to biotic stress were identified. It is noteworthy that $63 O N A C$ genes exhibited overlapping expression patterns in response to abiotic and biotic stresses and most of these ONAC genes were upregulated under abiotic (salt, drought, or cold) and biotic (infection by $M$. oryzae and infestation by S. hermonthica) stress (Figure 5F). Among these, ONAC026, ONAC069, ONAC095, ONAC108, and $O N A C 117$ were responsive to all the abiotic and biotic stresses, while $8 O N A C$ genes were responsive to most of the abiotic and biotic stresses (Figure 5E, Supplementary file 2).

\subsection{Evaluation Relationships of ONAC Genes with Overlapping Expression Patterns}

To clarify possible evaluation relationships, we constructed a phylogenetic tree for a total of $86 O N A C$ genes including 63 ONAC genes with overlapping expression patterns, several previously reported $O N A C$ genes, and some homologous $O N A C$ genes using aligned protein sequences. The resulted phylogenetic tree is similar to the unrooted tree constructed previously by Nuruzzaman et al. [15], which showed that the stress-responsive $O N A C$ genes can be divided into 10 groups (Figure 6). In our phylogenetic tree, 12 ONAC genes belong to the SNACII (NAM/CUC3) group, 7 ONAC genes 
belong to the ONAC11 (NAC1) group, 9 ONAC genes belong to the OsNAC7 (SND) group, 5 ONAC genes belong to the SNACIII (TIP) group, 3 ONAC genes belong to the OsNAC8 group, 12 ONAC genes belong to the SNACI group, 6 ONAC genes belong to the SNACIV (ANAC34) group, 10 ONAC genes belong to the ONAC012 (ONAC7) group, 11 ONAC genes belong to the ONAC100 (ONAC2) group, and 11 to the ONAC135 (ONAC3) group. These data suggested that these putative stress-responsive $O N A C$ genes show significant evolutional diversity and those $O N A C$ genes clustered in same group may have the same or similar biological functions. The SNACI group includes 12 known ONAC genes-SNAC1, OsNAC3, OsNAC4, OsNAC5, OsNAC6, ONAC010, ONAC016, ONAC058, $O N A C 088, O N A C 103, O N A C 122$, and ONAC131 - and most members of this group are responsive to different abiotic (e.g., salt, drought, and cold) and biotic (e.g., infection by M. oryzae and infestation by S. hermonthica) stresses (Figures 5 and 6 and Supplementary file 2). Particularly, 4 of them-ONAC058, $O N A C 103, O N A C 122$, and $O N A C 131$-were previously shown to be upregulated after infection by viral pathogens, RSV, or rice tungro spherical virus (RTSV) [15]. In the SNACII (NAM/CUC3) group, overexpression of ONACO45 in rice can improve drought and salt tolerance [31] and OsNAC1 and OSNAC2 were identified previously [58]. Importantly, 7 ONAC genes-ONAC026, ONAC039, ONAC045, ONAC092, ONAC107, ONAC123, and ONAC132-showed upregulated expression patterns in response to abiotic (e.g., salt, drought, and cold) and biotic (infection by M. oryzae or RSV) stresses (Figures 5 and 6 and Supplementary file 2). In the SNACIII (TIP) group, RIMI (ONAC054) has been demonstrated to be a negative regulator of defense response against rice dwarf virus [41]. RIM1, $O N A C 110$, and ONAC121 exhibited upregulated expression patterns under abiotic (e.g., salt, drought, and cold) and biotic (infection by M. oryzae) stresses, whereas ONAC109 showed downregulated expression under all the abovementioned stresses except RSV infection (Figures 5 and 6 and Supplementary file 2). In the SNAC IV (ANAC34) group, ONAC063 was shown to be inducible to high salt stress and overexpression in Arabidopsis increased salt tolerance [59]. ONAC96 and ONAC140 exhibited upregulated expression patterns under abiotic (e.g., salt, drought, and cold) and biotic (infection by $M$. oryzae or RSV) stresses (Figures 5 and 6 and Supplementary file 2). OsNAC8 is highly homologous to Arabidopsis NTL6, which has been shown to be a cold-responsive NAC gene with function in disease resistance response [60]. OsNAC8 exhibited upregulated expression patterns under abiotic (e.g., salt, drought, and cold) and biotic (infection by M. oryzae) stresses. Most of the ONAC genes, such as ONAC056, ONAC052, andONAC084 in the OSNAC7 (SND) group, were downregulated under salt and drought stresses (Figures 5 and 6 and Supplementary file 2), but were upregulated under cold and infection by RSV, Xoo, or Xoc (Figures 5 and 6 and Supplementary file 2). Expression of $O N A C 008$ and $O N A C 043$ in the ONAC011 (NAC1) group was upregulated in response to salt, drought, and cold and to infection by $M$. oryzae, whereas expression of $O N A C 043$ of this group was induced by $X O O$ and Xoc (Figures 5 and 6 and Supplementary file 2). ONAC028 and ONAC108, two members of the ONAC079 (ONAC6) group, were upregulated in response to salt and drought stress and to $X O C$ infection (Figures 5 and 6 and Supplementary file 2). ONAC012, ONAC059, and ONAC017 in the ONAC012 (ONAC7) group were induced by cold and M. oryzae, whereas ONAC012 and ONAC017 were induced by $X o O$ and $X o c$ (Figures 5 and 6 and Supplementary file 2). Most of the members in the ONAC135 (ONAC3) and ONAC100 (ONAC2) groups were responsive to biotic stresses (infection by M. oryzae, Xoo, Xoc, or RSV) (Figures 5 and 6 and Supplementary file 2), indicating their possible involvement in biotic stress response. 


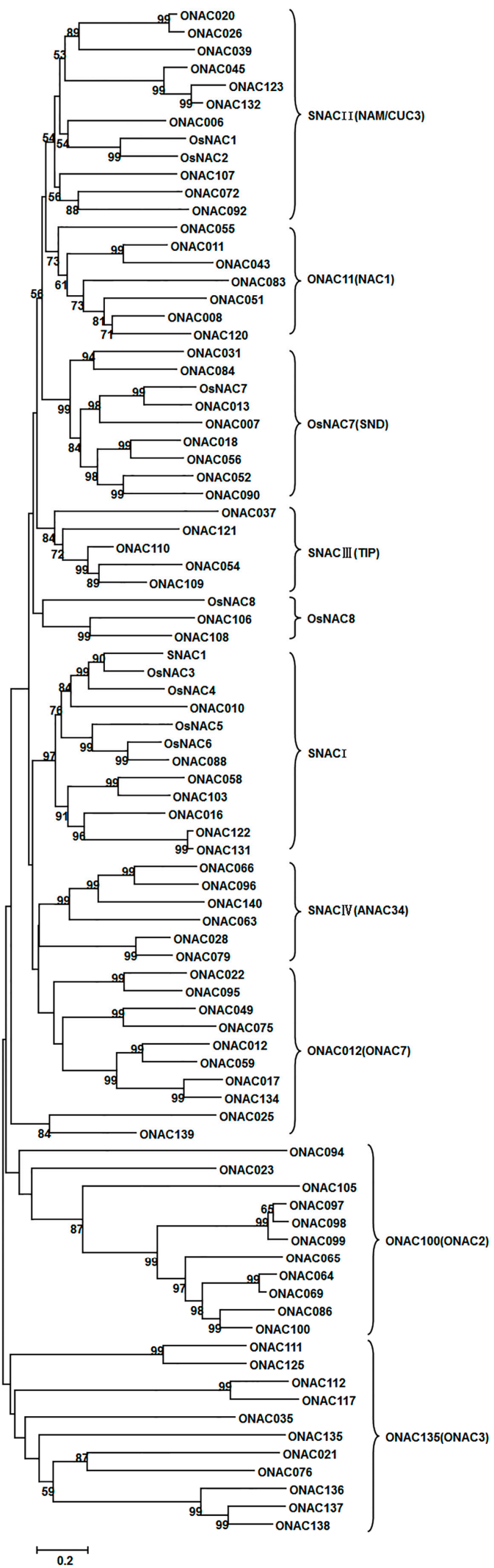

Figure 6. Uprooted phylogenetic tree representing relationships among stress-responsive $O N A C$ genes. Full-length protein sequences of 86 ONAC genes (63 ONAC genes showing overlapping expression patterns, several previously reported $O N A C$ genes, and some homologous $O N A C$ genes) were used for multiple alignments by the clustalw2 program and a phylogenic tree was created and visualized using MEGA 6. 


\section{Discussion}

In plants, the expression of stress-responsive genes is transcriptionally activated by different forms of abiotic stresses and by different pathogens. Overlapping expression of the stress-responsive genes is a result of coordination of plant responses to different abiotic and biotic stresses $[61,62]$. In the present study, we identified a total of 63 ONAC genes that showed overlapping expression patterns in rice seedlings/plants under different abiotic and biotic stresses from analyses of public microarray data. Most of these $O N A C$ genes with overlapping expression patterns (e.g., those belonging to the SNACI, SNACII, SNACIII, SNACIV, and ONAC011 groups) were upregulated in response to abiotic (salt, dry, and cold) and biotic (infection by M. oryzae and infestation by S. hermonthica) stresses. Among the 63 ONAC genes with overlapping expression patterns identified in our analysis, 7 ONAC genes-SNAC1, OsNAC3, OsNAC4, OsNAC5, OsNAC6, OsNAC045, and OsNAC10 (ONAC122) - were previously reported to be induced by drought, low temperature, or high salinity conditions [25-31], indicating the validity of our microarray data-based analysis of the ONAC gene expression under different abiotic and biotic stress. Our quantitative RT-PCR analysis of 30 selected $O N A C$ genes in rice seedlings after treatments with drought, salt, or cold stresses, or infection by M. oryzae or RSV, further confirmed the overlapping expression features of these $O N A C$ genes, showing similar or identical expression patterns to those obtained from analysis of the rice microarray data. ABA is a well-known stress hormone that mediates signaling pathways involved in defense response against abiotic and biotic stresses [50,51]. In the present study, 16 ONAC genes were upregulated in response to ABA. Previous studies have shown that SNAC1, OsNAC5, OsNAC6, ONAC045, and OsNAC10 were strongly induced by ABA [25,26,29-31]. These results indicate that these stress-responsive $O N A C$ genes might be involved in ABA hormone-mediated signaling pathways and thus play important roles in the stress response. Taken together, members of the ONAC family are responsive to multiple abiotic and biotic stresses and thus might play important roles in mediating defense response against different abiotic and biotic stresses. However, functional analysis using loss-of-function and gain-of-function mutants or transgenic lines is critical to elucidate the biological function of these ONAC genes with overlapping expression patterns in rice abiotic and biotic stress response.

Most of the $O N A C$ members identified in the present study were shown to be involved in responses to multiple stresses and stress-related signal molecule, and exhibited complicated overlapping expression patterns under different abiotic and biotic stress conditions. Previous studies have shown that members of the $O N A C$ genes may have pleiotropic function in tolerance against different abiotic stresses and defense response against biotic stress. For example, transgenic rice plants overexpressing OsNAC6 exhibited an improved tolerance to dehydration and high-salt stresses, and also exhibited increased tolerance to blast disease [26,28]. Similarly, overexpression of stress-responsive $S N A C 1$ or OsNAC5 enhanced drought and salt tolerance in rice and many stress-responsive genes were upregulated in the transgenic plants [25,29]. The responsiveness of these stress-responsive $O N A C$ genes with overlapping expression patterns under different abiotic and biotic stress conditions might imply that they have pleiotropic biological functions in the stress response, although detailed functional analysis using mutants and/or overexpression transgenic lines is required. The pleiotropic feature of the biological functions of the $O N A C$ genes makes them excellent candidates for efforts aimed at genetic improvement of stress tolerance in various kinds of plants including rice. 


\section{Methods}

\subsection{Plant Growth and Treatments}

Rice (Oryza sativa L.) subsp. indica cultivar Yuanfengzao was used in this study. Seeds were germinated and grown on soil (clay mixed with one-quarter soil) in a greenhouse with natural sunlight. Seedlings were moved to a growth chamber $\left(28{ }^{\circ} \mathrm{C} 14 \mathrm{~h}\right.$ light $>1000 \mu \mathrm{mol} \cdot \mathrm{m}^{-2} \cdot \mathrm{s}^{-1} / 26^{\circ} \mathrm{C} 10 \mathrm{~h}$ dark $)$ 3 days before the experiment.

For ABA treatment, 7-day-old seedlings were sprayed with $100 \mu \mathrm{M}$ ABA and kept for $3 \mathrm{~h}$. Salt stress was administered by transferring 7-day-old seedlings to a beaker containing $200 \mathrm{mM} \mathrm{NaCl}$ solution for $3 \mathrm{~h}$ at $28{ }^{\circ} \mathrm{C}$; drought stress was carried out by placing 7-day-old seedlings onto paper towels for $3 \mathrm{~h}$ at $28^{\circ} \mathrm{C}$; cold treatment was achieved by keeping 7-day-old seedlings of cv. Yuanfengzao at $4{ }^{\circ} \mathrm{C}$ for $3 \mathrm{~h}$. Seedlings kept at $28{ }^{\circ} \mathrm{C}$ for $3 \mathrm{~h}$ were used as a control. Samples were harvested after designated time points and stored at $-80^{\circ} \mathrm{C}$ until use.

For inoculation with $M$. oryzae, 2-week-old cv. Yuanfengzao seedlings were inoculated by foliar spraying with spore suspension $\left(5 \times 10^{5}\right.$ spores $/ \mathrm{ml}$ in $0.02 \%(v / v)$ Tween-20) of strain $85-14 \mathrm{~B} 1$ of the blast fungus (race ZB1) until uniformly covered with tiny droplets. Mock-inoculation controls were prepared by spraying with $0.02 \%(v / v)$ Tween-20. The inoculated and mock-inoculated seedlings were kept at $100 \%$ relative humidity in the dark for $24 \mathrm{~h}$ and then returned to the growth chamber. Leaf samples were collected at different time points (72 and $96 \mathrm{~h}$ after inoculation) and stored at $-80{ }^{\circ} \mathrm{C}$ until use. For inoculation with RSV, 9-day-old seedlings were infested by RSV-carrying Laodelphax striatellus insects for 2 days and then transferred to an insect-free chamber at $25 \pm 3{ }^{\circ} \mathrm{C}$ with a daily photoperiod. Control plants were not infested by insects but transferred to the same insect-free chamber. Leaf samples were harvested 8 days after infestation and stored at $-80{ }^{\circ} \mathrm{C}$ until use.

\subsection{Mining and Analysis of Microarray-Based Expression Profiling Data}

Microarray data publicly available on the Rice Oligo Array Database [63] were used in this study. Data from accessions GSE6901 (expression data for abiotic stress treatment), GSE7256 and GSE18361 (expression data for rice leaf and root infection with M. oryzae strains FR13 and Guy11, respectively), GSE16793 (expression data for interactions with Xoo or Xoc), GSE11025 (expression data for interaction with RSV), and GSE10373 (expression data for interaction with S. hermonthica) were mined and analyzed for expression patterns of the rice $O N A C$ genes. Information on the $O N A C$ genes and entire microarray experiments used in this study is listed in Supplementary file 4, 6 and 7 [49,52,53,55-57]. Probe sets for a total of $107 O N A C$ genes were included in the Affymetrix rice genome array and IDs of probe sets representing $O N A C$ genes were identified. The probe set of every representative $O N A C$ gene (Supplementary file 5) shows normalized signal intensities. Expression data for these 107 ONAC genes under different abiotic and biotic stress conditions were mined from the Rice Oligonucleotide Array database, and transferred as $\log _{2}{ }^{\text {signal intensity }}$ value for each probe ID corresponding to ONAC genes. A differentially expressed gene in a given abiotic or biotic stress was defined according to the value of $\log _{2}$ (signal intensity in treatment/signal intensity in control) $=\log _{2}{ }^{\text {signal intensity in treatment }}-\log _{2}$ signal intensity in control and upregulation or downregulation of $O N A C$ genes was chosen for the analysis when the value of $\log _{2}$ (signal intensity in treatment/signal intensity in control) is $>1$ or $<-1$ [15] and the relative expression folds is $>2$ or $<0.5$, 
respectively. The FDR level for detecting significant differential expression was set to 0.01 . Genes with a $p$-value of $<0.05$ were considered to be differentially expressed. Hierarchical clustering heat maps were generated by TM4 MultiExperiment Viewer (MeV), which calculates pairwise Euclidean distances using the average linkage method.

\section{3. qPCR Analysis}

For quantitative real-time PCR analysis, total RNA was extracted from frozen leaf samples with TRIzol (Invitrogen, Shanghai, China), according to the manufacturer's instructions. Resulting RNA samples were treated with RNase-free DNase (TaKaRa, Dalian, China) and first strand cDNAs were synthesized using AMV reverse transcriptase (TaKaRa, Dalian, China), following the manufacturer's instructions. QPCR reactions were performed as previously described [64]. Gene specific primers were synthesized for 30 ONAC genes and one actin gene (Os05g36290) and listed in Supplementary file 8. Expression data for each $O N A C$ gene under different abiotic and biotic conditions were normalized using the expression data of the actin gene as an internal reference. Relative expression level was calculated by the comparative $\Delta \Delta C_{\mathrm{t}}$ method [65]. The $\Delta C_{\mathrm{t}}$ and $\Delta \Delta C_{\mathrm{t}}$ were calculated by the formulas: $\Delta C_{\mathrm{t}}($ target gene $)=C_{\mathrm{t}}($ target gene $)-C_{\mathrm{t}}($ actin $)$ and $\Delta \Delta C_{\mathrm{t}}=\Delta C_{\mathrm{t}}($ different time points $)-\Delta C_{\mathrm{t}}(0 \mathrm{~h})$ or $\Delta \Delta C_{\mathrm{t}}=\Delta C_{\mathrm{t}}($ treated sample $)-\Delta C_{\mathrm{t}}($ untreated sample $)$. Expression folds for each $O N A C$ gene under a given stress were calculated by the formulas of fold $=2^{-\Delta \Delta C t}$. Standard errors of the means from three independent biological replicates were calculated.

\subsection{Phylogenetic Analysis}

Protein sequences for $86 O N A C$ genes (63 ONAC genes with overlapping expression patterns, several previously reported $O S N A C$ genes, and some $O N A C$ homologous genes, Supplementary file 3) were obtained from the Rice Genome Annotation [66] and were subject to phylogenetic analysis. The alignment was adjusted manually and unrooted phylogenetic trees were constructed by the neighbor-joining method using MEGA 6 software [67]. The confidence level of monophyletic groups was estimated using a bootstrap analysis of 1000 replicates. A phylogenetic tree was created, and visualized using MEGA 6 software.

\section{Conclusions}

In the present study, we analyzed the expression of $O N A C$ genes based on the rice microarray data and the results showed that a total of $63 O N A C$ genes showed overlapping expression patterns in rice seedlings/plants under various different abiotic and biotic stress conditions. These $O N A C$ genes with overlapping expression patterns might have pleiotropic biological functions in stress response. Results from this systematic analysis of the $O N A C$ gene family presented not only evidence for the possible role of $O N A C$ genes in response to abiotic and biotic stresses, but also provided clues for further functional analysis of $O N A C$ genes in stress tolerance and pathogen resistance. 


\section{Supplementary Materials}

Supplementary materials can be found at http://www.mdpi.com/1422-0067/16/02/4306/s1.

\section{Acknowledgments}

We thank Zuhua He, Institute of Plant Physiology and Ecology, Shanghai Institutes for Biological Sciences, Chinese Academy of Sciences, for providing the rice materials and Rongyao Chai, Zhejiang Academy of Agricultural Science, for providing the strain of M. oryzae and technical assistance in disease assays. This work was supported by the National Natural Science Foundation of China (Nos. 30971880 and 31400315), National R\&D Project of Transgenic Crops of the Ministry of Agriculture of China (No. 2011ZX08009-003-001), Doctoral degree of Education Ministry of China (No. 20120101110070), and the Natural Science Foundation of Jiangsu Province (No. BK20130389).

\section{Author Contributions}

Lijun Sun, Lei Huang, Yongbo Hong, Huijuan Zhang, and Fengming Song performed bioinformatics and gene expression analysis. Dayong Li contributed to data interpretation and wrote the manuscript with Lijun Sun.

\section{Conflicts of Interest}

The authors declare no conflict of interest.

\section{References}

1. Nakashima, K.; Yamaguchi-Shinozaki, K.; Shinozaki, K. The transcriptional regulatory network in the drought response and its crosstalk in abiotic stress responses including drought, cold, and heat. Front. Plant Sci. 2014, 5, 170, doi:10.3389/fpls.2014.00170.

2. Golldack, D.; Li, C.; Mohan, H.; Probst, N. Tolerance to drought and salt stress in plants: Unraveling the signaling networks. Front. Plant Sci. 2014, 5, 151, doi:10.3389/fpls.2014.00151.

3. Hines, P.J.; Zahn, L.M. What's bugging plants? Plant-microbe interactions. Introduction. Science 2009, 324, 741, doi:10.1126/science.324_741.

4. Boller, T.; He, S.Y. Innate immunity in plants: An arms race between pattern recognition receptors in plants and effectors in microbial pathogens. Science 2009, 324, 742-744.

5. Atkinson, N.J.; Urwin, P.E. The interaction of plant biotic and abiotic stresses: from genes to the field. J. Exp. Bot. 2012, 63, 3523-3543.

6. Golldack, D.; Lüking, I.; Yang, O. Plant tolerance to drought and salinity: Stress regulating transcription factors and their functional significance in the cellular transcriptional network. Plant Cell Rep. 2011, 30, 1383-1391.

7. Gutterson, N.; Reuber, T.L. Regulation of disease resistance pathways by AP2/ERF transcription factors. Curr. Opin. Plant Biol. 2004, 7, 465-471.

8. Eulgem, T.; Somssich, I.E. Networks of WRKY transcription factors in defense signaling. Curr. Opin. Plant Biol. 2007, 10, 366-371. 
9. Singh, K.; Foley, R.C.; Oñate-Sánchez, L. Transcription factors in plant defense and stress responses. Curr. Opin. Plant Biol. 2002, 5, 430-436.

10. Aida, M.; Ishida, T.; Fukaki, H.; Fujisawa, H.; Tasaka, M. Genes involved in organ separation in Arabidopsis: An analysis of the cup-shaped cotyledon mutant. Plant Cell 1997, 9, 841-857.

11. Ernst, H.A.; Olsen, A.N.; Larsen, S.; Lo Leggio, L. Structure of the conserved domain of ANAC, a member of the NAC family of transcription factors. EMBO Rep. 2004, 5, 297-303.

12. Duval, M.; Hsieh, T.F.; Kim, S.Y. Thomas, T.L. Molecular characterization of AtNAM: A member of the Arabidopsis NAC domain superfamily. Plant Mol. Biol. 2002, 50, 237-248.

13. Ooka, H.; Satoh, K.; Doi, K.; Nagata, T.; Otomo, Y.; Murakami, K.; Matsubara, K.; Osato, N.; Kawai, J.; Carninci, P.; et al. Comprehensive analysis of NAC family genes in Oryza sativa and Arabidopsis thaliana. DNA Res. 2003, 10, 239-247.

14. Fang, Y.; You, J.; Xie, K.; Xie, W.; Xiong, L. Systematic sequence analysis and identification of tissue-specific or stress-responsive genes of NAC transcription factor family in rice. Mol. Genet. Genomics 2008, 280, 547-563.

15. Nuruzzaman, M.; Manimekalai, R.; Sharoni, A.M.; Satoh, K.; Kondoh, H.; Ooka, H.; Kikuchi, S. Genome-wide analysis of NAC transcription factor family in rice. Gene 2010, 465, 30-44.

16. Rushton, P.J.; Bokowiec, M.T.; Han, S.; Zhang, H.; Brannock, J.F.; Chen, X.; Laudeman, T.W.; Timko, M.P. Tobacco transcription factors: Novel insights into transcriptional regulation in the Solanaceae. Plant Physiol. 2008, 147, 280-295.

17. Le, D.T.; Nisjiyama, R.; Watanabe, Y.; Mochida, K.; Yamaquchi-Shinozaki, K.; Shinozaki, K.; Tran, L.S. Genome-wide survey and expression analysis of the plant-specific NAC transcription factor family in soybean during development and dehydration stress. DNA Res. 2011, 18, 263-276.

18. Hu, R.; Qi, G.; Kong, Y.; Kong, D.; Gao, Q.; Zhou, G. Comprehensive analysis of NAC domain transcription factor gene family in Populus trichocarpa. BMC Plant Biol. 2010, 10, 145.

19. Olsen, A.N.; Ernst, H.A.; Leggio, L.L.; Skriver, K. NAC transcription factors: Structurally distinct, functionally diverse. Trends Plant Sci. 2005, 10, 79-87.

20. Zhong, R.; Lee, C.; Ye, Z.H. Evolutionary conservation of the transcriptional network regulating secondary cell wall biosynthesis. Trends Plant Sci. 2010, 15, 625-632.

21. Jiang, Y.; Deyholos, M.K. Comprehensive transcriptional profiling of NaCl-stressed Arabidopsis roots reveals novel classes of responsive genes. BMC Plant Biol. 2006, 6, 25.

22. Tran, L.S.; Nakashima, K.; Sakuma, Y.; Simpson, S.D.; Fujita, Y.; Maruyama, K.; Fujita, M.; Seki, M.; Shinozaki, K.; Yamaguchi-Shinozaki, K. Isolation and functional analysis of Arabidopsis stress inducible NAC transcription factors that bind to a drought responsive cis-element in the early responsive to dehydration stress 1 promoter. Plant Cell 2004, 16, 2481-2498.

23. Wu, Y.; Deng, Z.; Lai, J.; Zhang, Y.; Yang, C.; Yin, B.; Zhao, Q.; Zhang, L.; Li, Y.; Yang, C.; Xie, Q. Dual function of Arabidopsis ATAF1 in abiotic and biotic stress responses. Cell Res. 2009, 19, 1279-1290.

24. Fujita, M.; Fujita, Y.; Maruyama, K.; Seki, M.; Hiratsu, K.; Ohme-Takagi, M.; Tran, L.S.; Yamaguchi-shinozaki, K.; Shinozaki, K. A dehydration-induced NAC protein, RD26, is involved in a novel ABA-dependent stress-signaling pathway. Plant J. 2004, 39, 863-876. 
25. Hu, H.; Dai, M.; Yao, J.; Xiao, B.; Li, X.; Zhang, Q.; Xiong, L. Overexpressing a NAM, ATAF, and CUC (NAC) transcription factor enhances drought resistance and salt tolerance in rice. Proc. Natl. Acad. Sci. USA 2006, 103, 12987-12992.

26. Hu, H.; You, J.; Fang, Y.; Zhu, X.; Qi, Z.; Xiong, L. Characterization of transcription factor gene SNAC2 conferring cold and salt tolerance in rice. Plant Mol. Biol. 2008, 67,169-181.

27. Ohnishi, T.; Sugahara, S.; Yamada, T.; Kikuchi, K.; Yoshiba, Y.; Hirano, H.Y. Tsutsumi, N. OsNAC6, a member of the NAC gene family, is induced by various stresses in rice. Genes Genet. Syst. 2005, 80, 135-139.

28. Nakashima, K.; Tran, L.S.; Van Nguyen, D.; Fujita, M.; Maruyama, K.; Todaka, D.; Ito, Y.; Hayashi, N.; Shinozaki, K.; Yamaguchi-Shinozaki, K. Functional analysis of a NAC-type transcription factor $O S N A C 6$ involved in abiotic and biotic stress-responsive gene expression in rice. Plant J. 2007, 51, 617-630.

29. Takasaki, H.; Maruyama, K.; Kidokoro, S.; Ito, Y.; Fujita, Y.; Shinozaki, K.; Yamaguchi, S.K.; Nakashima, K. The abiotic stress-responsive NAC-type transcription factor OsNAC5 regulates stress-inducible genes and stress tolerance in rice. Mol. Genet. Genomics 2010, 284, 173-183.

30. Jeong, J.S.; Kim, Y.S.; Baek, K.H.; Jung, H.; Ha, S.H.; Do Choi, Y.; Kim, M.; Reuzeau, C.; Kim, J.K. Root-specific expression of OsNAC10 improves drought tolerance and grain yield in rice under field drought conditions. Plant Physiol. 2010, 153, 185-197.

31. Zheng, X.; Zhen, B.; Lu, G.; Han, B. Overexpression of a NAC transcription factor enhances rice drought and salt tolerance. Biochem. Biophys. Res. Commun. 2009, 379, 985-989.

32. Peng, H.; Yu, X.; Cheng, H.; Shi, Q.; Zhang, H.; Li, J.; Ma, H. Cloning and characterization of a novel NAC family gene CarNAC1 from chickpea (Cicer arietinum L.). Mol. Biotechnol. 2010, 44, $30-40$.

33. Peng, H.; Cheng, H.Y.; Chen, C.; Yu, X.W.; Yang, J.N.; Gao, W.R.; Shi, Q.H.; Zhang, H.; Li, J.G.; Ma, H. A NAC transcription factor gene of chickpea (Cicer arietinum L.), CarNAC3, is involved in drought stress response and various developmental processes. J. Plant Physiol. 2009, 166, 1934-1945.

34. Peng, H.; Cheng, H.Y.; Yu, X.W.; Shi, Q.H.; Zhang, H.; Li, J.G.; Ma, H. Characterization of a chickpea (Cicer arietinum L.) NAC family gene, CarNAC5, which is both developmentally and stress-regulated. Plant Physiol. Biochem. 2009, 47, 1037-1045.

35. Yang, R.; Deng, C.; Ouyang, B.; Ye, Z. Molecular analysis of two salt-responsive NAC-family genes and their expression analysis in tomato. Mol. Biol. Rep. 2011, 38, 857-863.

36. Han, Q.; Zhang, J.; Li, H.; Luo, Z.; Ziaf, K.; Ouyang, B.; Wang, T.; Ye, Z. Identification and expression pattern of one stress-responsive NAC gene from Solanum lycopersicum. Mol. Biol. Rep. 2011, 39, 1713-1720.

37. Wang, X.; Basnayake, B.M.; Zhang, H.; Li, G.J.; Li, W.; Virk, N.; Menqiste, T.; Song, F. The Arabidopsis ATAF1, a NAC transcription factor, is a negative regulator of defense responses against necrotrophic fungal and bacterial pathogens. Mol. Plant Microbe. Interact. 2009, 22, 1227-1238.

38. Delessert, C.; Kazan, K.; Wilsom, L.W.; van der Straetend, D.; Manners, J.; Dennis, E.S.; Dolferus, R. The transcription factor ATAF2 represses the expression of pathogenesis-related genes in Arabidopsis. Plant J. 2005, 43, 745-757. 
39. Jensen, M.K.; Rung, J.H.; Gregersen, P.L.; Gjetting, T.; Fuglsang, A.T.; Hansen, M.; Joehnk, N.; Lyngkjaer, M.F.; Collinge, D.B. The HvNAC6 transcription factor: A positive regulator of penetration resistance in barley and Arabidopsis. Plant Mol. Biol. 2007, 65, 137-150.

40. Kaneda, T.; Taga, Y.; Takai, R.; Iwano, M.; Matsui, H.; Takayama, S.; Isoga, A.; Che, F.S. The transcription factor OsNAC4 is a key positive regulator of plant hypersensitive cell death. EMBO J. 2009, 28, 926-936.

41. Yoshii, M.; Shimizu, T.; Yamazaki, M.; Higashi, T.; Miyao, A.; Hirochika, H.; Omura, T. Disruption of a novel gene for a NAC-domain protein in rice confers resistance to rice dwarf virus. Plant J. 2009, 57, 615-625.

42. Ren, T.; Qu, F.; Morris, T.J. HRT gene function requires interaction between a NAC protein and viral capsid protein to confer resistance to turnip crinkle virus. Plant Cell 2000, 12, 1917-1926.

43. Xie, Q.; Sanz-Burgos, A.P.; Guo, H.; García, J.A.; Gutiérrez, C. GRAB proteins, novel members of the NAC domain family, isolated by their interaction with a geminivirus protein. Plant Mol. Biol. 1999, 39, 647-656.

44. Selth, L.A.; Dogra, S.C.; Rasheed, M.S.; Healy, H.; Randles, J.W.; Rezaian, M.A. A NAC domain protein interacts with tomato leaf curl virus replication accessory protein and enhances viral replication. Plant Cell 2005, 17, 311-325.

45. Oh, S.K.; Lee, S.; Yu, S.H.; Choi, D. Expression of a novel NAC domain-containing transcription factor $(\mathrm{CaNACl})$ is preferentially associated with incompatible interactions between chili pepper and pathogens. Planta 2005, 222, 876-887.

46. Xia, N.; Zhang, G.; Liu, X.Y.; Deng, L.; Cai, G.L.; Zhang, Y.; Wang, X.J.; Zhao, J.Z.; Huang, L.L.; Kang, Z.S. Characterization of a novel wheat NAC transcription factor gene involved in defense response against stripe rust pathogen infection and abiotic stresses. Mol. Biol. Rep. 2010, 37, 3703-3712.

47. Faria, J.A.; Reis, P.A.; Reis, M.T.; Rosado, G.L.; Pinheiro, G.L.; Mendes, G.C.; Fontes, E.P. The NAC domain-containing protein, GmNAC6, is a downstream component of the ER stressand osmotic stress-induced NRP-mediated cell-death signaling pathway. BMC Plant Biol. 2011, 11, 129, doi:10.1186/1471-2229-11-129.

48. Mahajan, S.; Tuteja, N. Cold, salinity and drought stresses: An overview. Arch. Biochem. Biophys. 2005, 444, 139-158.

49. Tyagi, A.K.; Kapoor, S.; Khurana, J.P.; Ray, S. Expression data for stress treatment in rice seedlings. 2007, GSE6901. Rice Array Database. Available online: http://www.ricearray.org (accessed on 15 October 2014).

50. Kim, T.H. Plant stress surveillance monitored by ABA and disease signaling interactions. Mol. Cells 2012, 33, 1-7.

51. Wasilewska, A.; Vlad, F.; Sirichandra, C.; Redko, Y.; Jammes, F.; Valon, C.; Frei dit Frey, N.; Leung, J. An update on abscisic acid signaling in plants and more. Mol. Plant 2008, 1, 198-217.

52. Balzergue, S.; Morel, J.; Martin-Magnetite, M.L. Identification of rice genes differentially expressed upon Virulent Infection by Magnaporthe oryzae. 2007, GSE7256. Rice Array Database. Available online: http://www.ricearray.org (accessed on 15 October 2014). 
53. Marcel, S.; Sawers, R.; Oakeley, E.; Angliker, H.; Paszkowski, U. Temporal gene expression analyisis from rice root (cv. Nipponbare) infected with Magnaporthe oryzae strain Guy11. 2009, GSE18361. Rice Array Database. Available online: http://www.ricearray.org (accessed on 15 October 2014).

54. Lin, R.M.; Zhao, W.S.; Meng, X.B.; Wang, M.; Peng, Y.L. Rice gene OsNAC19 encodes a novel NAC-domain transcription factor and responds to infection by Magnaporthe grisea. Plant Sci. 2007, 172, 120-130.

55. DO, N.L. Comparative transcriptional profiling of rice undergoing infection by X. oryzae pv. oryzae or by X. oryzae pv.oryzicola. 2009, GSE16793. Rice Array Database. Available online: http://www.ricearray.org (accessed on 15 October 2014).

56. Zhang, X.; Wu, Z.; Xie, L.; Lin, Q.; Xie, L. Comparative transcriptional profiling of two contrasting rice genotypes in response to rice stripe virus infection. 2008, GSE11025. Rice Array Database. Available online: http://www.ricearray.org (accessed on 15 October 2014).

57. Scholes, J.D.; Swarbrick, P.J.; Huang, K.; Slate, J.; Press, M.C. Rice cultivars undergoing a susceptible and resistant interaction with the parasitic Plant STRIGA Hermonthica. 2008, GSE10373. Rice Array Database. Available online: http://www.ricearray.org (accessed on 15 October 2014).

58. Kikuchi, K.; Ueguchi-Tanaka, M.; Yoshida, K.T.; Nagato, Y.; Matsusoka, M.; Hirano, H.Y. Molecular analysis of the NAC gene family in rice. Mol. Gen. Genet. 2000, 262, 1047-1051.

59. Yokotani, N.; Ichikawa, T.; Kondou, Y.; Matsui, M.; Hirochika, H.; Iwabuchi, M.; Oda K. Tolerance to various environmental stresses conferred by the salt-responsive rice gene ONAC063 in transgenic Arabidopsis. Planta 2009, 229, 1065-1075.

60. Seo, P.J.; Kim, M.J.; Park, J.Y.; Kim, S.Y.; Jeon, J.; Lee, Y.H.; Kim, J.; Park, C.M. Cold activation of a plasma membrane-tethered NAC transcription factor induces a pathogen resistance response in Arabidopsis. Plant J. 2010, 61, 661-671.

61. Cheong, Y.H.; Chang, H.S.; Gupta, R.; Wang, X.; Zhu, T.; Luan, S. Transcriptional profiling reveals novel interactions between wounding, pathogen, abiotic stress, and hormonal responses in Arabidopsis. Plant Physiol. 2002, 129, 661-677.

62. Fujita, M.; Fujita, Y.; Noutoshi, Y.; Takahashi, F.; Narusaka, Y.; Yamaguchi-Shinozaki, K.; Shinozaki, K. Crosstalk between abiotic and biotic stress responses: A current view from the points of convergence in the stress signaling networks. Curr. Opin. Plant Biol. 2006, 9, 436-442.

63. Rice Oligonucleotide Array Database. Available online: http://www.ricearray.org/ (accessed on 15 October 2014).

64. Sun, L.; Zhang, H.; Li, D.; Huang, L.; Hong, Y.; Ding, X.S.; Nelson, R.S.; Zhou, X.; Song, F. Functions of rice NAC transcriptional factors, ONAC122 and ONAC131, in defense responses against Magnaporthe grisea. Plant Mol. Biol. 2013, 81, 41-56.

65. Livak, K.J.; Schmittgen, T.D. Analysis of relative gene expression data using real-time quantitative PCR and the $2^{-\Delta \Delta C t}$ method. Methods 2001, 25, 402-408. 
66. Rice Genome Annotation Project. Available online: http://rice.plantbiology.msu.edu/ (accessed on 15 October 2014).

67. Tamura, K.; Stecher, G.; Peterson, D.; Filipski, A.; Kumar, S. MEGA6: Molecular Evolutionary Genetics Analysis version 6.0. Mol. Biol. Evol. 2013, 30, 2725-2729.

(C) 2015 by the authors; licensee MDPI, Basel, Switzerland. This article is an open access article distributed under the terms and conditions of the Creative Commons Attribution license (http://creativecommons.org/licenses/by/4.0/). 